EGU2020-1893

https://doi.org/10.5194/egusphere-egu2020-1893

EGU General Assembly 2020

(c) Author(s) 2020. This work is distributed under

the Creative Commons Attribution 4.0 License.

\title{
Test of Dust Emission Over the Middle East
}

Mokhammad Suleiman Mostamandi ${ }^{1}$, Georgiy Stenchikov ${ }^{1}$, Alexander Ukhov ${ }^{1}$, Illia Shevchenko ${ }^{1}$, Johann Engelbrecht ${ }^{2}$, Yasser Alshehri ${ }^{1}$, and Anatolii Anisimov ${ }^{1,3}$

${ }^{1}$ KAUST, Earth Science and Engineering, Thuwal - Jeddah, Saudi Arabia (suleiman.mostamandy@gmail.com)

${ }^{2}$ Desert Research Institute, Reno, Nevada, USA

${ }^{3}$ Marine Hydrophysical Institute, Russian Academy of Sciences, Sevastopol, Russia

\begin{abstract}
The dust emission simulated within the up-to-date global and regional models differs by almost an order of magnitude. The models are tuned to reproduce the observed aerosol optical depth (AOD) that, with some caveats, reflects the dust mass retained in the atmosphere. However, the amount of dust suspended in the atmosphere is controlled independently by the dust emission and deposition; therefore, only AOD observations are insufficient to constrain both these processes. To calculate the dust emission over the Middle East (ME), in this study, we employ dust deposition observations, AERONET AOD, micro-pulse lidar, and satellite observations to constrain the WRFChem simulations. The dust deposition is measured on a monthly bases for 2015-2019 using passive samplers over six sites over land and the sea. We compare the WRF-Chem simulations, conducted with $10-\mathrm{km}$ grid spacing, with the recent MERRA-2 and CAMS reanalysis. WRF-Chem is configured with the GOCART dust scheme. We calculate the meteorological and aerosol initial and boundary conditions using the MERRA-2 reanalysis.
\end{abstract}

We evaluated the dust regional mass balance controlled by emission, deposition, and crossboundary transport. The smallest dust particles are transported at vast distances while the heaviest ones deposit inside of the domain. Since the model accounts for dust particles with radii $<10$ um, we process the deposition samples to extract the weight of particles smaller than 10 um. WRF-Chem was tuned to reproduce the observed AOD and monthly deposition of dust particles with radii $<10 \mathrm{um}$. We found that the ME dust emission comprises about $30 \%$ of the global annual dust emission. MERRA-2 underestimates the ME dust emission by about $15 \%$. 